\title{
Platelet Lysate and Non-Healing Ulcers
}

\author{
Alexander Lykov ${ }^{1,2, *} \mathbb{D}$, Maria Surovtseva ${ }^{1,2} \mathbb{D}$, Natalia Bondarenko $\left.{ }^{1,2} \mathbb{(}\right)$, Irina Kim ${ }^{1,2} \mathbb{D}$, Mikhail \\ Smagin 1(i), Olga Poveshchenko ${ }^{1,2}$ (i) \\ 1 Research Institute of Clinical and Experimental Lymphology-Branch of the Institute of Cytology and Genetics Siberian \\ Branch of the Russian Academy of Sciences (RICEL-Branch ICG SB RAS), Novosibirsk, Russia \\ 2 Meshalkin National Medical Research Center (MNMRC), Ministry of Health of Russian Federation, Novosibirsk, Russia \\ * Correspondence: aplykov2@mail.ru;
}

Scopus Author ID 7005078523

Received: 5.12.2020; Revised: 30.12.2020; Accepted: 2.01.2021; Published: 3.01.2021

\begin{abstract}
Platelet lysate contained a wide range of bioactive molecules involved in cell proliferation, migration. The paper analyzes the possibility of using platelet lysate for the treatment of non-healing ulcers. The levels of bioactive molecules were determined by ELISA in platelet lysate. Efficacy of treatment with platelet lysate patients with diabetic foot ulcers, ulcers in patients with peripheral arterial disease and venous insufficiency were analyzed on wound surface area, total epithelialization. Twice injection of platelet lysate with an interval of 5-7 days possess to wound surface area closure on Day 180 at $96 \%$ in patients with diabetic foot ulcers, while in patients with peripheral arterial disease and venous insufficiency ulcers wound closure was less. Platelet lysate from patients with non-healing ulcers contained growth factor, cytokine, components of the extracellular matrix, and nitric oxide. Between levels of bioactive molecules in platelet lysate and outcome were found significant correlation. The obtained results indicate that platelet lysate possesses wound healing activity.
\end{abstract}

Keywords: non-healing wound; platelet lysate; growth factor; components of extracellular matrix; wound surface area; epithelialization.

(C) 2020 by the authors. This article is an open-access article distributed under the terms and conditions of the Creative Commons Attribution (CC BY) license (https://creativecommons.org/licenses/by/4.0/).

\section{Introduction}

Wound healing is a complex process, including inflammation, angiogenesis, and extracellular matrix formation for damaged tissue repair. Different types of cells, growth factors, and cytokines play a crucial role in regulating wound healing. Disruption in healing processes may delay repair at the wound site and lead to chronic non-healing wound formation [1].

Peripheral arterial disease, venous insufficiency, and diabetes mellitus caused chronic non-healing foot ulcer formation (trophic ulcer) [2-5]. Non-healing foot ulcers' tendency to relapse and expand, resistance to traditional treatment, expensive, deterioration of quality of life, disability, and mortality are reasons for searching for new treatment methods, including autologous platelet-rich plasma (PRP).

PRP is often used in regenerative medicine to promote tissue healing as an alternative source of growth factors and cytokines involved in the regeneration of damaged tissues [6]. The main source of growth factor and cytokines in platelets is $\alpha$ granules, including plateletderived growth factor (PDGF), transforming growth factor- $\beta$ (TGF- $\beta$ ), vessel endothelial growth factor (VEGF), fibroblast growth factor (FGF), insulin-like growth factor-1 (IGF1), and epidermal growth factor (EGF) [7, 8]. 
Previously we demonstrated that platelet-poor plasma (PPP) and PRP from patients with diabetic foot syndrome (SDF) significantly decreased human dermal fibroblast, human bone marrow mesenchymal stem cells, and endothelial cells line EA.Hy926 proliferation and migration capacities compared with PPP and PRP from patients with venous non-healing wounds [9]. Also, we demonstrated that the combination of traditional methods of treating patients with non-healing wounds with autologous PRP possesses wound epithelization activity [10].

Thus, it is of interest to compare the therapeutical potential of platelet lysate (PL) in treating non-healing wound repair in patients with DFS and foot ulcers of PAD and venous insufficiency.

\section{Materials and Methods}

\subsection{Patients.}

The study was conducted with respect to the Helsinki declaration's principles. The informed consent is received from all participants. Based on the laboratory of cellular technology of the RICEL-Branch ICG SB RAS and MNMRC, and based on the Department of Vascular Surgery of the RICEL-Branch ICG SB RAS in 2019-2020, we examined and treated 30 patients with non-healing ulcers: 19 patients with DFU and 11 patients with PAD and venous insufficiency. For patients with ulcers of more than 4-weeks duration, located on foot (plantar, medial, or lateral surface), wound area range from $0,5 \mathrm{~cm}^{2}$ to $75 \mathrm{~cm}^{2}$ was included. All patients were divided into 4 groups according to the methods of treatment: 1 traditional methods of treatment in patients with DFU; 2- patients with DFU treated with PL; 3 - traditional methods of treating patients with PAD and venous insufficiency; 4 - patient with PAD and venous insufficiency treatment with PL. The patients with DFU range from 49 to 81 years, ranging from 65 to 82 years in patients with PAD and venous insufficiency, and was not statistically different across the comparison groups.

\subsection{Platelet lysate preparation.}

Plasma was received by sedimentation of blood in the special tubes (Plasmolifting ${ }^{\mathrm{TM}}$, Russia) containing thixotropic gel and sodium heparin during 6-8 minutes at $3800 \mathrm{rpm}$ on EBA200 centrifuge (Hettich, Germany) (3-4 tubes from each patient), further, after the transfer of plasma in new tubes it was repeatedly centrifuged at $3500 \mathrm{rpm}$ during 15 minutes on LMC 3000 centrifuge (Biosan, Latvia), collected supernatant plasma (PPP), to prepare PL pellet platelets was resuspended in $1 \mathrm{ml}$ of PPP and underwent to twice cycle freeze/thaw, then passed through MF-Millipore ${ }^{\mathrm{TM}}$ filter (Merck, USA), packed in sterile vials. One vial was used immediately for treatment, and 2 vials were stored at $-70^{\circ} \mathrm{C}$. Then $5-7$ days later, the $2^{\text {nd }}$ vial of PL was thawed at $37^{\circ} \mathrm{C}$ and used for treatment. The $3^{\text {rd }}$ vial with PL was used for ELISA.

\subsection{Treatment procedure.}

In all patients, non-healing ulcers were cleaned from necrotic and infected tissues. Then, patients received traditional treatment include antibiotics, necrotomy, correction of glycemic levels, lipoic acid, and sulodexide. Additional patients of groups 2 and 4 received 3$4 \mathrm{~mL}$ PL injections, twice within an interval of 5-7 days. Primary outcome parameters, such as size (length and width) and wound surface area, were examined at the end of hospitalization, 3 
and 6 months late. The percentage of epithelization of the wound of the ulcers measured using a visual score.

\subsection{ELISA assays.}

Human insulin-like growth factor -1, Human Free Brain-Derived Neurotrophic Factor (R\&D systems), Total Collagen, Human Vimentin, and Human Fibronectin (Abcam), Human SDF-1a (CXCL 12A) (Thermo Scientific), Human ICAM-1 (eBioscience), TNF- $\alpha$, VEGF, and EPO (Vector-best) levels in PL were determined in duplicate according to manufacture's instructions.

\subsection{Nitrite levels.}

Nitric oxide (NO) levels in PL were measured using Griess reagent for nitrite determination (Merck, USA). The levels of NO were calculated using a sodium nitrite standard curve.

\subsection{Statistical analysis.}

Data were analyzed by the Statistica 10.0 for Windows. In this study, the normality of the distribution was determined by the w-Shapiro-Wilkes criterion. In tables, the obtained data were presented as mean \pm standard deviation $(\mathrm{SD})$, the data were analyzed by one-way analysis of variance (ANOVA) with a Bonferroni correction (Bonferroni post hoc test) to analyze differences between groups. If the p-value was less than 0.05 , it was considered statistically significant. In this study, the correlation coefficient was determined using the Spearman rank correlation (Rs).

\section{Results and Discussion}

\subsection{Effect of platelet lysate treatment of non-healing ulcer outcomes.}

We enrolled in this study, 30 patients with non-healing ulcers occurred in patients with DFU and patients with PAD and venous insufficiency. In groups of patients who received PL treatment, it should be noted that high-frequency patients with a large wound surface area. In control groups of patients with non-healing ulcers which received traditional treatment frequency of wound surface area more than $3 \mathrm{~cm}^{2}$ among patients with DFU occurred in $40 \%$ (range from $4 \mathrm{~cm}^{2}$ to $35 \mathrm{~cm}^{2}$ ), and among patients with PAD and venous insufficiency in $67 \%$ (range from $4 \mathrm{~cm}^{2}$ to $15 \mathrm{~cm}^{2}$ ). While among patients with non-healing ulcers received PL treatment frequency of wound surface area more than $3 \mathrm{~cm}^{2}$ in patients with DFU observed in $64 \%$ (range from $4 \mathrm{~cm}^{2}$ to $30 \mathrm{~cm}^{2}$ ) and patients with PAD and venous insufficiency in $80 \%$ (range from $4 \mathrm{~cm}^{2}$ to $12 \mathrm{~cm}^{2}$ ). Wound surface area in patients from Group 2 was significantly large compare with Group 1 on the day of enrollment to study. At the end of the study (Day 180), we observed significantly increased wound surface area in Group 1 compared with Group 2 (Fig. $1 ; \mathrm{p}<0.05$ ). Any differences in wound surface area among non-healing ulcers in patients with PAD and venous insufficiency was not observed during this study (Fig. 1; p>0.05). Also, we did not find any significant difference in wound surface area among patients with DFU on Day 10, and Day 90 (Figure 1; p>0.05), while on Day 180, wound surface area in Group 4 was significantly large than in Group $2(\mathrm{p}<0.05)$. 


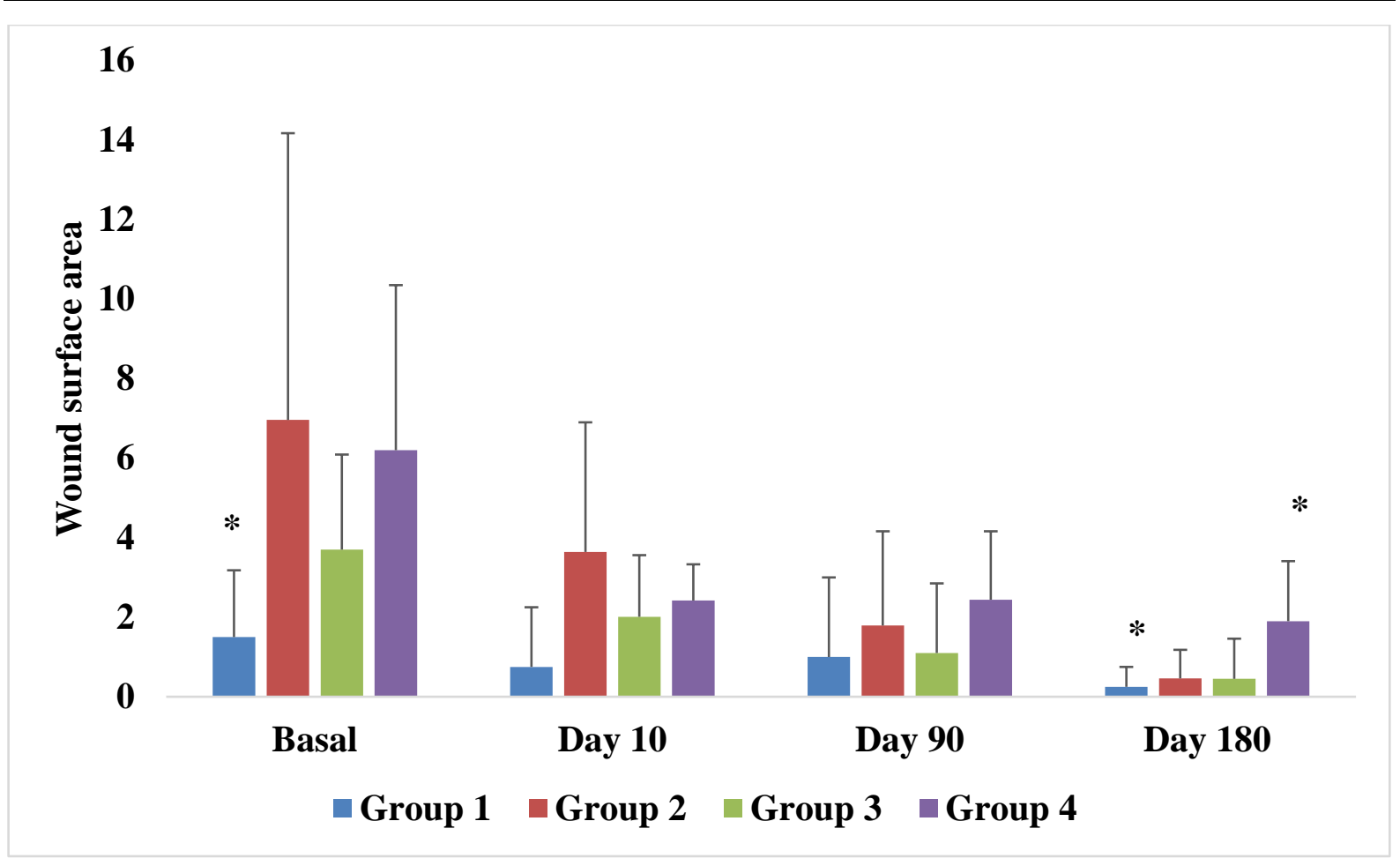

Figure 1.Wound surface area changes in patients with non-healing ulcers. p<0,05*with Group 2.

The therapeutic potential of PL in non-healing ulcers can be assessed by wound surface area epithelialization. In patients from Group 1, the rate of epithelialization of non-healing ulcers achieved 80\% (Figure 2). Whereas in Group 2, at the end of the study, the wound surface area's epithelialization reached $96 \%$. In Group 3, traditional treatment possesses epithelialization of $87 \%$ of wound surface area at Day 180. Whereas in Group 4 was observed a decreased rate of wound surface epithelialization at the end of the study, there was a relapse and expansion of non-healing ulcers in one of the patients.

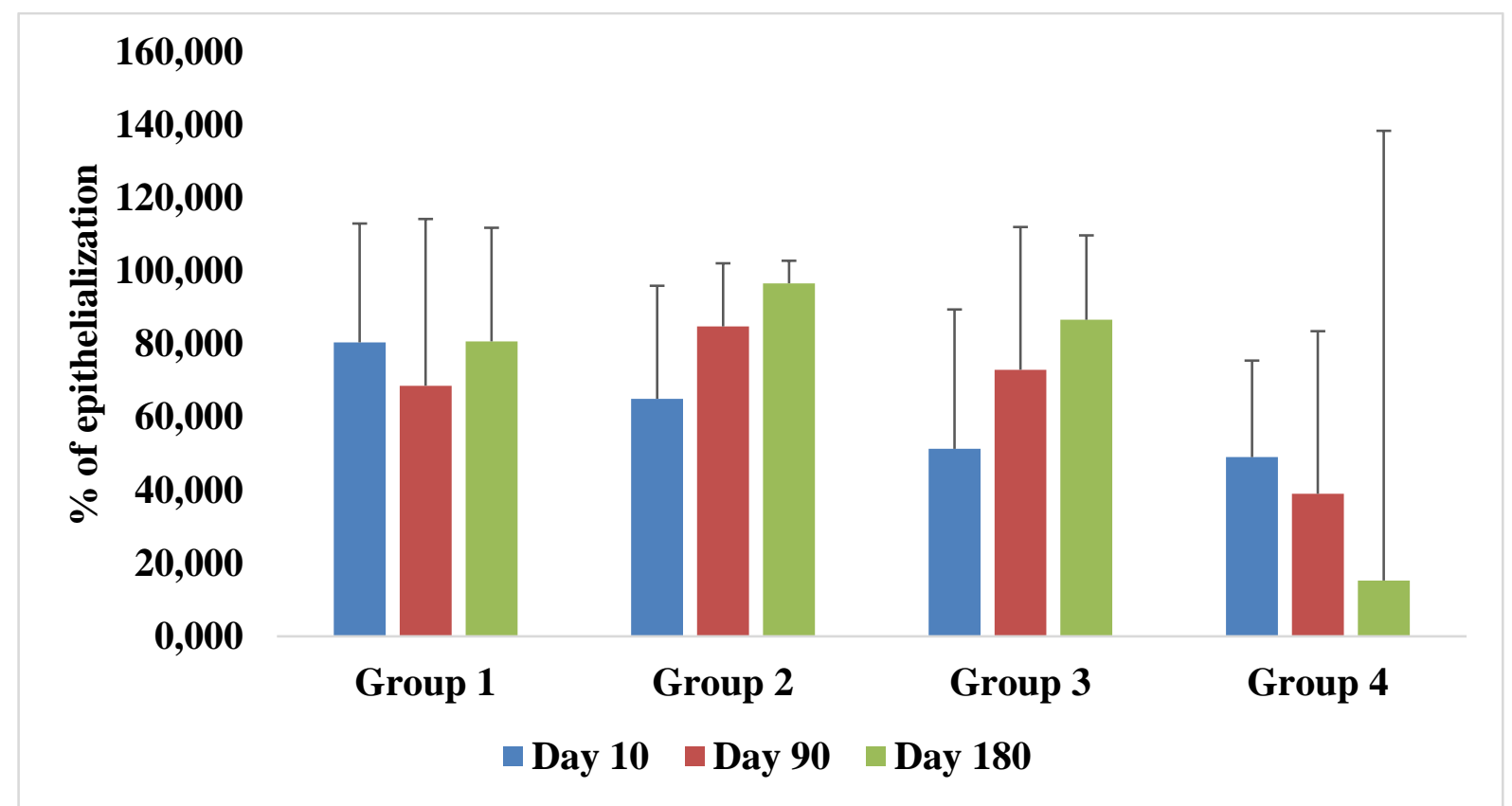

Figure 2.Effect of traditional treatment and platelet lysate treatment in patients with non-healing ulcers on epithelialization rate.

Authors under meta-analysis of PRP's therapeutic efficacy in the treatment of DFU patients wrote about a statistically significant benefit [11]. Rainys and coworkers studied the 
effect of autologous PRP gel in the treatment of hard-to-heal leg ulcers. They wrote about the increased frequency of completely re-epithelialization and wound size reduction [12]. Joshi and coworkers estimated that PRP treatment possesses complete wound healing in patients with a pressure ulcer [13]. The therapeutic potential of dressing with PRP was studied to treat clean non-healing DFU and estimated that reduced wound surface area and time to maximum healing [14]. Authors reported PRP's therapeutical potential in patients with trophic ulcers in leprosy, which possess to decrease wound surface area [15]. On the basis of PRP treatment of 38 patients with DFU, authors wrote about total epithelialization of wound surface on day 80 [16]. Everts and coworkers wrote about different PRP formulations used for wound healing [17]. Our study used the modification of commonly prepared PRP characterized in that we do not activate platelets but subject them to destruction during freezing/thawing cycles, which also contributes to the release of biologically active molecules from platelets. In addition, by filtering the PL, we remove debris and reduce the risk of stimulating an inflammatory response in the wound defect. For example, for treatment of wounds used leukocytes platelet-rich fibrin [18]. Combination of non-activated leukocyte-rich PRP with nano-fat grafting possess to achieve complete wound healing in $53.8 \%$ of patients [19]. Gentile and coworkers compared the therapeutic potential of autologous human activated and non-activated PRP [20].

\subsection{Bioactive molecules profile of platelet lysate from patients with non-healing ulcers.}

Platelet number in PRP from patients with PAD and venous insufficiency enrolled in Group 4 was significantly lower compared within the same parameter in Group 2, and Group 3 (Figure 3; $\mathrm{p}$ 0.05). We have not obtained the presence of platelets in PL after 2 cycles of freeze/thaw and filtration from all patients with non-healing ulcers enrolled in the study.

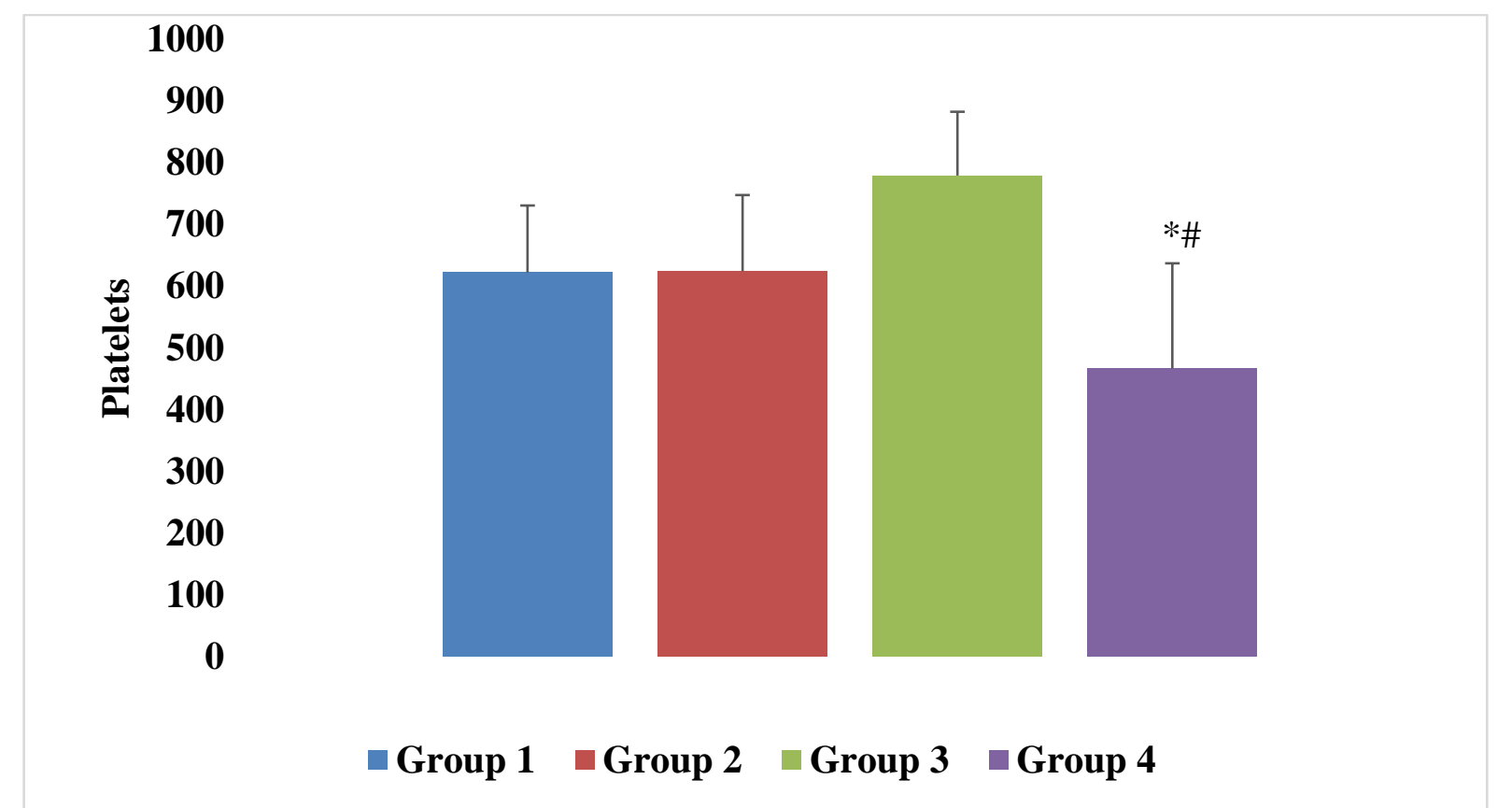

Figure 3.The number of platelets $\left(10^{6} / \mathrm{mL}\right)$ in platelet-rich plasma of patients with non-healing ulcers. $\mathrm{p}<0.05$ *with Group 2, \#with Group 3.

ELISA was used to screen for bioactive molecules present in PL. We have determined the presence of growth factors (EPO, VEGF, IFG-1, BDNF, SDF-1 $\alpha$ ), TNF- $\alpha$, extracellular matrix components (Vimentin, Fibronectin, Collagen), ICAM-1, and NO in PL obtained from patients with non-healing ulcers (Table 1). The levels of bioactive molecules in PL from 
patients with non-healing ulcers have no have differences, while the levels of VEGF in PL from Group 2 was significantly higher. The levels of NO were significantly lower than Group 1 $(\mathrm{p}<0.05)$, and EPO levels, ICAM-1 in PL of Group 2 were significantly lower compared with Group 4.

Table 1. The levels of bioactive molecules in platelet lysate.

\begin{tabular}{c|c|c|c|c} 
Parameters & Group 1 & Group 2 & Group 3 & Group 4 \\
\hline EPO, $\mathrm{mIU} / \mathrm{mL}$ & $64.99 \pm 12.29$ & $66.62 \pm 15.23$ & $85.9 \pm 20$ & $95.9 \pm 18.4 \#$ \\
\hline VEGF, pg/mL & $604.1 \pm 29.9$ & $658.8 \pm 57.5^{*}$ & $597.9 \pm 48.5$ & $589.3 \pm 126.6$ \\
\hline IGF-1, $\mathrm{ng} / \mathrm{mL}$ & $4.31 \pm 3.18$ & $4.65 \pm 1.77$ & $5.3 \pm 3$ & $4.1 \pm 1$ \\
\hline BDNF, pg/mL & $1315.6 \pm 314.7$ & $1113 \pm 271,9$ & $1200.4 \pm 440.3$ & $1080 \pm 678.9$ \\
\hline SDF-1a, $\mathrm{pg} / \mathrm{mL}$ & $1633.3 \pm 108.9$ & $1778.4 \pm 264.6$ & $1607 \pm 74.2$ & $1640 \pm 117.1$ \\
\hline TNF- $\alpha, \mathrm{pg} / \mathrm{mL}$ & $139.6 \pm 11.5$ & $143.5 \pm 18.8$ & $130.5 \pm 1.5$ & $134.6 \pm 6.7$ \\
\hline $\mathrm{NO}, \mu \mathrm{M} / \mathrm{mL}$ & $19.4 \pm 3.8$ & $14.5 \pm 3.7 *$ & $17.1 \pm 3.7$ & $16.7 \pm 2.9$ \\
\hline Vimentin, $\mathrm{ng} / \mathrm{mL}$ & $122.3 \pm 7,9$ & $127.6 \pm 11.53$ & $126.1 \pm 15.4$ & $131.9 \pm 5.6$ \\
\hline Fibronectin, $\mathrm{pg} / \mathrm{mL}$ & $4430,3 \pm 1017$ & $4417.2 \pm 760$ & $4076.3 \pm 1126.3$ & $4177.4 \pm 886.7$ \\
\hline Collagen, $\mu \mathrm{g} / 10 \mu \mathrm{LL}$ & $6.44 \pm 1.48$ & $4.43 \pm 1.76^{*}$ & $4.2 \pm 1.9$ & $3.4 \pm 1.1$
\end{tabular}

Note. EPO, erythropoietin; VEGF, vessel endothelial growth factor; IGF-1, insulin-like growth factor; BDNF, brain-derived growth factoe; SDF-1a, stromal-derived factor-1 alpha; TNF- $\alpha$, tumor necrosis factor-alpha; NO, nitric oxide; ICAM-1, inter-cell adhesion molecule-1. p<0.05*with Group 1, \#with Group 2.

Gad and coworkers compared PRP and sildenafil (an angiogenesis stimulator) treatment in experimental skin wound healing in rats. They estimated that both components decreased systemic proinflammatory cytokine levels [21]. A comparison of PRP obtained from patients with and without diabetes revealed that patients with diabetes had higher VEGF levels [22]. Platelet lysate is considered a replacement option for fetal calf serum for cell expansion $[23,24,25]$. Platelet lysate has been shown to contain growth factors, cytokines, and other biologically active molecules necessary for cell growth and proliferation. Delila and coworkers showed that PL contained IGF-1 [24]. Yan and coworkers showed that PL contained PDGFAA, IGF-1, TGF- $\beta$, EGF, and FGF, which are involved in therapeutic effects during wound healing [26].

3.3. Correlation between outcome and bioactive molecules in platelet lysate from patients with non-healing ulcers.

There were found negative strong correlations between age of patients from Group 1 with TNF- $\alpha$ and IGF-1 levels in PL $(\mathrm{Rs}=-0.97, \mathrm{p}<0.05)$, and strong positive correlation between platelete number with Vimentin levels in PL $(R s=0.97, p<0.05)$. There were found positive correlation between age of pation from Group 2 with platelete number (Rs=0.69; $\mathrm{p}<0.05)$, Fibronectin levels in PL ( $R s=0.77 ; \mathrm{p}<0.05)$, and negative correlation with VEGF levels in PL (Rs=-0.59; $\mathrm{p}<0.05)$. Also we found correlations between platelete number and Fibronectin levels in PL (Rs=0.59; $<0.05)$, and negative correlation with IGF-1 in PL (Rs=$0.62 ; \mathrm{p}<0.05)$, and wound surface area $(\mathrm{Rs}=-0.62, \mathrm{Rs}=-0.61, \mathrm{Rs}=-0,6$ and $\mathrm{Rs}=-0.56, \mathrm{p}<0,05$ respectivelly basal, on Day 10, on Day 90 and on Day 180). Moreover, basal wound surface area correlate with IGF-1 and Collagen levels in PL $(\mathrm{Rs}=0.66$, and Rs=-0.65, p $<0.05$ respectivelly), wound surface area on Day 10 correlates with Fibronectin, IGF-1 and Collagen levels in PL ( $R s=-0.58, R s=0.58$, and $R s=-0.56$; $\mathrm{p}<0.05$ respectivelly).

In Group 3 we found a strong correlation between the age of patients with BDNF and VEGF levels in PL ( $R s=-0.98$, and $R s=0.89 ; \mathrm{p}<0.05$ respectively). Also, we found a correlation between platelet number and VEGF in PL $(R s=-0.89$; $p<0.05)$, between NO levels in PL with basal wound surface area (Rs=0.97; $<<0.05)$, and between EPO levels in PL with wound surface area on Day $90(\mathrm{Rs}=0.89 ; \mathrm{p}<0.05)$. In Group 4 we found correlation between platelet 
number with Vimentin in PL (Rs=-0.89; $\mathrm{p}<0.05)$, between Fibronectin levels in PL with basal wound surface area $(\mathrm{Rs}=-0,89 ; \mathrm{p}<0.05)$, between Vimentin and ICAM-1 levels in PL with wound surface are $4 a$ on Day 10 ( $R s=0,91$, and $R s=-0.89 ; p<0.05$ respectively), between wound surface area on Day 90 with BDNF, Collagen, and SDF- $1 \alpha$ in PL (Rs=-0.89; $\mathrm{p}<0.05)$, and between wound surface area on Day 180 with Fibronectin in Pl $(R s=0.89 ; p<0.05)$.

\section{Conclusions}

This study demonstrated that platelet lysate contained growth factors (such as EPO, VEGF, IFG-1, BDNF, SDF-1 $\alpha$ ), TNF- $\alpha$, extracellular matrix components (Vimentin, Fibronectin, Collagen), ICAM-1, and NO, which had correlations with outcome in patients with non-healing ulcers treated with platelet lysate. This treatment possesses to wound surface area epithelialization.

\section{Funding}

This research was carried out within the framework of government research assignment of RICEL-Branch of ICG SB RAS “Development of molecular profiling methods and innovative technologies for prediction, early diagnosis, drug and cell therapy of socially significant human diseases of autoimmune, inflammatory and dysmetabolic nature" No. AAAA-A19119031590016-0.

\section{Acknowledgments}

The authors thank Ekatherina Lykova for the article grammar correction.

\section{Conflicts of Interest}

The authors have no conflict of interest to declare.

\section{References}

1. Demidova-Rice, T.N.; Hamblin, M.R.; Herman, I.M. Acute and Impaired Wound Healing: Pathophysiology and Current Methods for Drug Delivery, Part 2Role of Growth Factors in Normal and Pathological Wound Healing Therapeutic Potential and Methods of Delivery. Adv. Skin Wound Care 2012, 25, https://doi.org/10.1097/01.ASW.0000418541.31366.a3.

2. Brouwer, R.J.; Lalieu, R.C.; Hoencamp, R.; van Hulst, R.A.; Ubbink, D.T. A systematic review and metaanalysis of hyperbaric oxygen therapy for diabetic foot ulcers with arterial insufficiency. J. Vasc. Surg. 2020, 71, 682-692, https://doi.org/10.1016/j.jvs.2019.07.082.

3. Bolton, L. Exercise and Chronic Wound Healing. Wounds: a Compendium of Clinical Research and Practice 2019, 31, 65-67.

4. Lin, C.; Liu, J.; Sun, H. Risk factors for lower extremity amputation in patients with diabetic foot ulcers: A meta-analysis. PLoS One 2020, 15, e0239236, https://doi.org/10.1371/journal.pone.0239236.

5. Yang, Y.; Chu, W.L.; Feng, G.; Zhang, H.J.; Hao, D.F. Retrospective analysis of 2997 inpatients with skin and soft tissue injuries. Zhonghua Shao Shang za zhi= Zhonghua Shaoshang Zazhi= Chinese Journal of Burns 2020, 36, 821-829, https://doi.org/10.3760/cma.j.cn501120-20200202-00036.

6. Etulain, J. Platelets in wound healing and regenerative medicine. Platelets 2018, 29, 556-568, https://doi.org/10.1080/09537104.2018.1430357.

7. Alves, R.; Grimalt, R. A Review of Platelet-Rich Plasma: History, Biology, Mechanism of Action, and Classification. Skin Appendage Disorders 2018, 4, 18-24, https://doi.org/10.1159/000477353.

8. Kaushik, A.; Kumaran, M. Platelet-rich plasma: The journey so far ! Indian Dermatology Online Journal 2020, 11, 685-692, https://doi.org/10.4103/idoj.IDOJ_369_19.

9. Lykov, A.P.; Surovtseva, M.A.; Poveshchenko, O.V.; Bondarenko, N.A.; Kim, I.I.; Yankaite, E.V. Evaluation of the Effect of Plasma from Patients with Trophic Ulcers on the Function of Dermal Fibroblasts, Mesenchymal Stem Cells, and Endothelial Cells. Bull. Exp. Biol. Med. 2020, 169, 558-563, https://doi.org/10.1007/s10517-020-04929-z. 
10. Smagin, M.A.; Shumkov, O.A.; Soluianov, M.I.; Demura, A.U.; Smagin, A.A.; Lykov, A.P.; Nimaev, V.V., Treatment of torpid trophic ulcers in patients of the older age group. Adv. Gerontol. 2020, 33, 373-378, https://doi.org/10.34922/AE.2020.33.2.022.

11. del Pino-Sedeño, T.; Trujillo-Martín, M.M.; Andia, I.; Aragón-Sánchez, J.; Herrera-Ramos, E.; Iruzubieta Barragán, F.J.; Serrano-Aguilar, P. Platelet-rich plasma for the treatment of diabetic foot ulcers: A metaanalysis. Wound Repair Regen. 2019, 27, 170-182, https://doi.org/10.1111/wrr.12690.

12. Rainys, D.; Cepas, A.; Dambrauskaite, K.; Nedzelskiene, I.; Rimdeika, R. Effectiveness of autologous platelet-rich plasma gel in the treatment of hard-to-heal leg ulcers: a randomised control trial. J. Wound Care 2019, 28, 658-667, https://doi.org/10.12968/jowc.2019.28.10.658.

13. Joshi, A.D.; More, S.N.; Mhambre, A.S. Treatment of a Chronic, Non-healing Neuropathic Ulcer in a Pediatric Patient With Spinal Dysraphism: A Case Report. Wound Manag Prev 2020, 66, 29-33.

14. Elsaid, A.; El-Said, M.; Emile, S.; Youssef, M.; Khafagy, W.; Elshobaky, A. Randomized Controlled Trial on Autologous Platelet-Rich Plasma Versus Saline Dressing in Treatment of Non-healing Diabetic Foot Ulcers. World J. Surg. 2020, 44, 1294-1301, https://doi.org/10.1007/s00268-019-05316-0.

15. Saha, S.; Patra, A.C.; Gowda, S.P.; Mondal, N.; Rahaman, S.; Ahmed, S.K.S.; Debbarma, S.; Vitthal, K.P.K.; Sarkar, S.; Sil, A. Effectiveness and safety of autologous platelet-rich plasma therapy with total contact casting versus total contact casting alone in treatment of trophic ulcer in leprosy: An observer-blind, randomized controlled trial. Indian Journal of Dermatology, Venereology, and Leprology 2020, 86, 262, https://doi.org/10.4103/ijdvl.IJDVL_571_18.

16. Duzhiy, I.D.; Nikolaienko, A.S.; Popadynets, V.M.; Kravets, O.V.; Hresko, I.Y.; Holubnichyi, S.O.; Sikora, V.V.; Lyndin, M.S.; Romaniuk, A.M. Reparative Processes Features in Trophic Ulcers Caused by Diabetes Mellitus With The Use Of Platelet-Rich Plasma. Wiadomosci Lekarskie (Warsaw, Poland: 1960) 2020, 73 , 2150-2155.

17. Everts, P.; Onishi, K.; Jayaram, P.; Lana, J.F.; Mautner, K. Platelet-Rich Plasma: New Performance Understandings and Therapeutic Considerations in 2020. Int. J. Mol. Sci. 2020, 21, 7794, https://doi.org/10.3390/ijms21207794.

18. Crisci, A.; Benincasa, G.; Crisci, M.; Crisci, F. Leukocyte Platelet-Rich Fibrin (L-PRF), a new biomembrane useful in tissue repair: basic science and literature review. Biointerface Research in Applied Chemistry 2018, 8, 3635-3643, https://doi.org/10.3390/diseases6030030.

19. Segreto, F.; Marangi, G.F.; Nobile, C.; Alessandri-Bonetti, M.; Gregorj, C.; Cerbone, V.; Gratteri, M.; Caldaria, E.; Tirindelli, M.C.; Persichetti, P. Use of platelet-rich plasma and modified nanofat grafting in infected ulcers: Technical refinements to improve regenerative and antimicrobial potential. Arch Plast Surg 2020, 47, 217-222, https://doi.org/10.5999/aps.2019.01571.

20. Gentile, P.; Calabrese, C.; De Angelis, B.; Dionisi, L.; Pizzicannella, J.; Kothari, A.; De Fazio, D.; Garcovich, S. Impact of the Different Preparation Methods to Obtain Autologous Non-Activated Platelet-Rich Plasma (A-PRP) and Activated Platelet-Rich Plasma (AA-PRP) in Plastic Surgery: Wound Healing and Hair Regrowth Evaluation. Int. J. Mol. Sci. 2020, 21, 431, https://doi.org/10.3390/ijms21020431.

21. Gad, S.B.; Hafez, M.H.; El-Sayed, Y.S. Platelet-rich plasma and/or sildenafil topical applications accelerate and better repair wound healing in rats through regulation of proinflammatory cytokines and collagen/TGF$\beta 1$ pathway. Environmental Science and Pollution Research 2020, 27, 40757-40768, https://doi.org/10.1007/s11356-020-10042-5.

22. Karina, K.; Wahyuningsih, K.A.; Sobariah, S.; Rosliana, I.; Rosadi, I.; Widyastuti, T.; Afini, I.; Wanandi, S.I.; Soewondo, P.; Wibowo, H.; Pawitan, J.A. Evaluation of platelet-rich plasma from diabetic donors shows increased platelet vascular endothelial growth factor release. Stem Cell Investigation 2019, https://doi.org/10.21037/sci.2019.10.02.

23. Barro, L.; Burnouf, P.-A.; Chou, M.-L.; Nebie, O.; Wu, Y.-W.; Chen, M.-S.; Radosevic, M.; Knutson, F.; Burnouf, T. Human platelet lysates for human cell propagation. Platelets 2020, https://doi.org/10.1080/09537104.2020.1849602.

24. Delila, L.; Wu, Y.-W.; Nebie, O.; Widyaningrum, R.; Chou, M.-L.; Devos, D.; Burnouf, T. Extensive characterization of the composition and functional activities of five preparations of human platelet lysates for dedicated clinical uses. Platelets 2020, https://doi.org/10.1080/09537104.2020.1849603.

25. Cao, B.; Dai, X. Platelet lysate induces chondrogenic differentiation of umbilical cord-derived mesenchymal stem cells by regulating the lncRNA H19/miR-29b-3p/SOX9 axis. FEBS Open Bio 2020, 10, 2656-2665, https://doi.org/10.1002/2211-5463.13002.

26. Yan, L.; Zhou, L.; Yan, B.; Zhang, L.; Du, W.; Liu, F.; Yuan, Q.; Tong, P.; Shan, L.; Efferth, T. Growth factors-based beneficial effects of platelet lysate on umbilical cord-derived stem cells and their synergistic use in osteoarthritis treatment. Cell Death Dis. 2020, 11, 857, https://doi.org/10.1038/s41419-020-03045-0. 